\title{
THE DIAGNOSIS OF ACUTE OSTEOMYELITIS OF THE PELVIS
}

\author{
Alan Morgan, \\ M.B., Ch.B. (Brist.), F.R.C.S. Eng.* \\ The Royal Infirmary \\ Alan K. Yates, \\ M.B., Ch.B. (Sheff.), F.R.C.S. Eng.** \\ Children's Hospital, Sheffield.
}

OSTEOMYELITIS of the pelvis has a sparse bibliography, probably because of its relative infrequency in general orthopaedics.

Froener (1889) stated that less than $10 \%$ of cases occured in the pelvis in a series of 545 cases of acute osteomyelitis; Butler (1940) gives an incidence of $8 \%$ in 500 cases of acute osteomyelitis seen at the London Hospital.

Von Bergmann (1906) found 63 cases of osteomyelitis of the ilium in 71 cases of osteomyelitis of the pelvis. Krasnobajiv (1925), Simmons (1915), Bearse (1923), Flickinger (1927) and Buosanti (1924) each give an incidence of osteomyelitis of the ilium of between $2 \%$ and $7 \%$. Young (1934), in his review of osteomyelitis of the ilium, to which he added three cases of his own, stated that "acute osteomyelitis of the pubis and ischium is so rare it is hardly necessary to give it consideration". His paper is the last comprehensive review of pelvic osteomyelitis, but referred only to the ilium.

This paper concerns the diagnosis of acute osteomyelitis of the pelvis in 18 patients seen in the Orthopaedic Departments of the Royal Infirmary and Childrens' Hospital in Sheffield between 1948 and 1964. Thirty case records of pelvic osteomyelitis were found among 616 cases of acute osteomyelitis of all bones seen during this period, but only 18 were documented sufficiently well for study. This gives an incidence of $5 \%$ of osteomyelitis occurring in the pelvis. Osteitis pubis due to surgery or trauma has not been included. Although this condition is more common than acute haematogenous osteomyelitis of the pelvis it is a separate entity. The principles of treatment of pelvic osteomyelitis do not differ from those of osteomyelitis in other bones and will not be discussed.

\section{Anatomy}

The incidence of involvement of individual bones of the pelvic ring in this series of 18 cases is as follows: Ilium, 14; Ischium, 4; Pubis, 0; Total, 18. This refers to the initial

\footnotetext{
Present address:

*United Cardiff Hospitals, Llandough Hospital, Cardiff.

**Guy's Hospital, London, S.E.1.
}

bone involved and does not include other bones of the pelvis involved by subsequent spread of the disease.

In agreement with other authors we find that the ilium is most commonly affected. The ilium forms the largest portion of the bony pelvis. Its blade has an abundant blood supply with a large nutrient artery entering its inner surface, and in the adult it is the only bone of the pelvis containing haemopoetic marrow.

The ischium was more commonly involved than in other series, but in no patient was the pubis primarily infected, although it was involved by extension in several cases. (Fig. 1).

\section{Clinical Presentation}

Although the correct diagnosis was made ultimately the condition often eluded diagnosis during weeks or even months of investigation.

On studying the case reports it became evident that certain features could be grouped into clinical syndromes which were related in general to the anatomical site of infection.

Most patients initially had symptoms and signs of toxaemia but did not come to hospital until localising signs, such as a limp, appeared some days later. A few patients suffered from septicaemia for a long period while the infective nidus remained hidden.

The following is a suggested classification based on our observations: -

1. Septicaemic

2. Clinical Syndromes

(a) Hip joint Syndrome.

(b) Abdominal Syndrome.

(c) Buttock Syndrome.

(d) Sciatic Syndrome.

\section{Septicaemic}

These patients have symptoms and signs of toxaemia, usually with a positive blood culture, but with no obvious infective nidus. Careful examination of the skeleton is essential in all cases of unexplained septicaemia. It is pertinent that eight of our patients had initial septicaemic symptoms on admission.

The following case history is illustrative.

Case no. 1. J.S. aged 31 years. Admitted initially under medical care with a pyrexia of unknown origin. He had been ill for one week with malaise, pyrexia and rigors. There was no relevant antecedent 


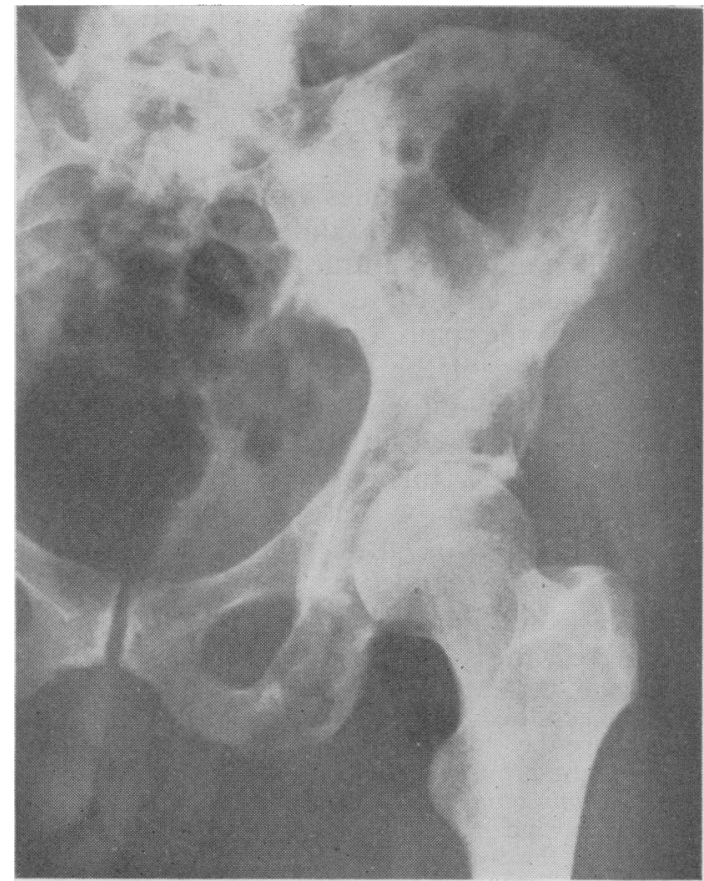

FIG. 1.-Radiograph of case J.S. showing bone destruction in the ilium at the anterior inferior iliac spine spreading to the acetabular roof, pubis and ischium.

history and no abnormal physical signs were noted. Initial investigations showed a white cell count of $19,500 / \mathrm{cu}$. mm.; $86 \%$ polys. Blood culture grew a pure growth of staphylococcus pyogenes.

Antibiotic treatment was commenced and was continued for eight weeks with varying combinations of drugs without success. Blood cultures remained positive with increasing resistance of the staphylococcus. Extensive investigations were carried out in an unsuccessful attempt to find the source of the infection. It is interesting to note that an intravenous pyelogram performed at this time would have revealed the osteomyelitic focus in the left ischium if this area had not been obscured by a gonadal radiation screen. Nine weeks after admission the patient started to complain of pain in the left hip and groin. Radiography showed an osteomyelitic lesion in the left ischium. A deep buttock abscess developed and he was transferred to orthopaedic care. The abscess was drained and a hip spica applied. However, the infection progressed and Fig. 1 shows the extensive bony destruction spreading into the pubis and acetabular rool. The hip is fusing spontaneously.

\section{Clinical Syndromes}

Patients may present certain clinical features which indicate the diagnosis and the site of the osteomyelitis. Four syndromes can be defined. (a) Hip Joint Syndrome

This syndrome has clinical features like those of septic arthritis of the hip but with certain exceptions. The pain is felt in the hip and in

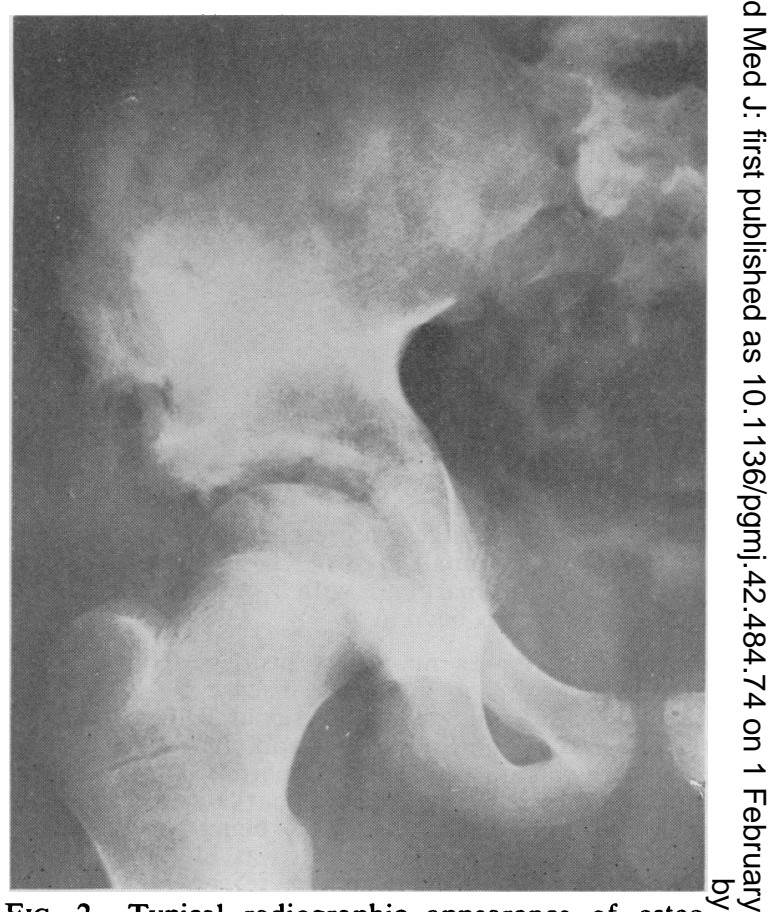

FIG. 2.-Typical radiographic appearance of osteomyelitis of the ilium, with separation of
sequestrum and spread to the acetabular roof

only one case was the pain also referred to the knee. Young (1934), states that there is no knee reference in osteomyelitis of the ilium. In general this may be true but the case reported below was exceptional in our series.

In agreement with Butler (1940) the commonest initial bony focus was in the region of the hip joint, especially the anterior iliac spines (Figs. 1, 2 and 3). One would expect to find tenderness at this point on examination and where this was specifically looked for, this was so. However, the focus in one case was in the ischium close to the acetabulum.

The main distinguishing feature of this lesion from purulent arthritis, where hip movement is limited in all directions, is a differential limitation of hip movement. Typically there is some degree of fixed flexion and loss of full internal rotation, but free movement in other directions.

Ten of our patients showed this picture and the following typical record is reported in detail: -

Case no. 2. A.L. aged 3 years. This child was admitted with a three day history of pain in the left hip and knee. He had been limping from the onset of the pain, but by the time of admission was unable to walk. He was hot and shivering and generally 


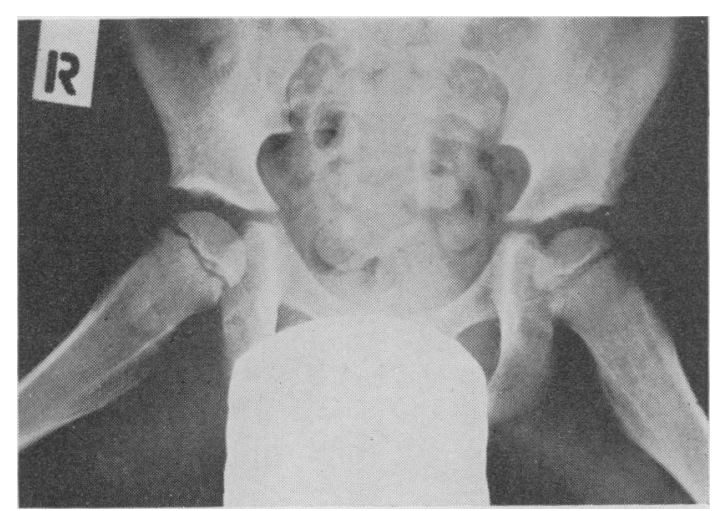

FiG. 3.-Early changes on radiography in case A.L. showing the small area of erosion just above the acetabular margin, with periosteal reaction extending up beyond it.

unwell. There was no recent history of sore throat or contact with any infectious disease.

On examination, he was pyrexial with a temperature of $39^{\circ} \mathrm{C}$. The throat, ears and chest were normal and there was no rash. The left hip was held in fixed flexion. Internal rotation was markedly reduced, the other movements at the hip being only slightly restricted. The white cell count was $21,000 / \mathrm{cu}$. mm., ESR $50 \mathrm{~mm}$./hr. Blood culture set up on admission grew a pure growth of coagulase positive staphylococcus pyogenes whioh was sensitive to penicillin. Aspiration of the hip joint yielded no pus.

The leg was placed on straight skin traction and systemic penicillin treatment commenced. On this regime the symptoms improved rapidly and the temperature subsided. Over the next three or four days, however, some tenderness developed over the body of the ilium but this subsided simultaneously with improvement of hip movements.

A radiograph twelve days after the onset of the symptoms showed some erosion of the bone just above the rim of the acetabulum with a periosteal reaction extending upwards from this point (Fig. 3). Only then was the diagnosis of osteomyelitis made.

Three weeks later, all the symptoms had completely settled. Penicillin was given for a total of six weeks. Six months later, the hip was clinically normal and nothing abnormal could be detected on further radiography.

\section{(b) Abdominal Syndrome}

This syndrome has some clinical features similar to those of acute appendicitis on the right side and paracolic abscess on the left. The patient was often referred to a general surgeon complaining of pain in one or other iliac fossa. In this series, all had disease on the right side and four had a palpable mass in the right iliac fossa. Two of these patients were subjected to laparotomy before the correct diagnosis was made. Weld (1960) described a case of osteomyelitis of the ilium which was initially diagnosed as acute appendicitis and had a normal appendix removed and Bruckner (1952) twice removed a normal appendix in similar circumstances.
There were other features which indicated the diagnosis of osteomyelitis of the pelvis. Five patients had the features of the hip syndrome and one of them had a buttock syndrome. (see below).

Appendicitis sometimes presents with psoas irritability. These cases differ from osteomyelitis by having early central abdominal pain and the hip signs are those of fixed flexion with increase of the pain on passive extension or hyperextension of the hip joint.

The initial bony focus in this group is usually situated in the blade of the ilium. This erodes the inner cortex, forming the abscess deep to iliacus which can be felt per abdomen. The oedematous iliacus itself also contributes to the size of the mass.

Six cases fell into this group and the following typical record is reported as an example.

Case no. 3. S.M. aged 20 years. This patient was initially admitted as a general surgical emergency with a five-day history of pyrexia and pain initially in the right groin and later in the right iliac fossa.

On examination there was tenderness in the right iliac fossa and fixed flexion with limited internal rotation of the hip. A diagnosis of acute appendicitis with psoas irritation was made but at operation a normal appendix was removed. Pain persisted in the right groin and the hip became very stiff, there being only ten degrees of movement in any direction. An area of tender induration appeared in the region of the greater trochanter and anterior superior iliac spine. A revised diagnosis of osteomyelitis of the head of the femur was made. Radiographs at this time showed no bony abnormality. Blood analysis revealed a white cell count of $7,000 / \mathrm{cu}$. $\mathrm{mm}$. with a normal differential count. ESR $45 \mathrm{~mm}$. $/ \mathrm{hr}$. A blood culture taken seven days after appendicectomy yielded a heavy growth of coagulase positive staphylococcus pyogenes sensitive to streptomycin, tetracycline and erythromycin but resistant to penicillin.

The leg was placed on skin traction on a Thomas' splint, changed later to an abduotion frame. The initial antibiotic treatment of penicillin was changed to streptomycin when blood culture sensitivities became available.

Fever and hip irritability persisted for four weeks during which time the infection extended from the ilium into the hip joint and pubis. Radiographs revealed complete obliteration of the hip joint space and the hip was immobilised in a spica for a further six months. The final picture two years later was of painful ankylosis requiring operative arthrodesis.

\section{(c) Buttock Syndrome}

This syndrome had separate clinical features of buttock pain, sometimes with radiation down the back of the thigh down to the level of the knee. Hip movements were typically free in the early stages but when the ischium was the site of the infective focus there was pain on abduction of the hip.

The initial bony focus was either in the posterior part of the iliac crest or the ischial 
tuberosity and the outer cortex of bone was eroded with an abscess enlarging into the overlying soft tissues.

Physical examination revealed a localised area of tenderness often with a palpable mass in the region of the infection. These findings made early diagnosis with accurate localisation of the infective focus possible. In our series these cases were correctly diagnosed at the time of first examination.

Six cases had clinical features of this group, and the following is a typical record.

Case no. 4. J.H. aged 15 years. The patient complained of increasing right buttock pain for six days with general malaise. There was no history of recent trauma.

On examination, the patient had a fever of $38.6^{\circ} \mathrm{C}$. There was free movement of the hips but some discomfort was experienced on flexion and abduction of the right hip and exquisite tenderness was present over the ischial tuberosity. Laboratory investigations revealed: a normal haemoglobin; WBC normal but with a relative polymorphonucleocytosis. ESR 43 $\mathrm{mm}$./hr. Blood culture revealed a heavy growth of coagulase positive staphylococcus pyogenes sensitive to penicillin.

Treatment was by leg traction and intramuscular penicillin. The patient was afebrile in seven days and the local symptoms and signs had completely resolved in eleven days. The patient was discharged home on the sixteenth day with no abnormal signs. Radiographs had appeared normal and a proven diagnosis had not been established.

Four months later a sinus developed in relation to the ischial tuberosity which now showed signs of bony infection on radiography. The patient persistantly refused operative treatment and after a prolonged course of antibiotics the sinus permanently healed with no residual disability one year later.

\section{(d) Sciatic Syndrome}

Only one case occurred in this series, but similar cases have been described in the literature. The syndrome is characterised by pain along the distribution of the sciatic nerve associated with the systemic signs of a purulent infection and localised tenderness over the region of the sacro-iliac joint. This is indicative of suppurative arthritis of the sacro-iliac joint. Avila (1941) described seven cases in whom these features were constant. Rendle Short (1931) described a similar case.

Suppurative arthritis of the sacro-iliac joint is probably secondary to osteomyelitis situated posteriorly in the blade of the ilium, the infection spreading later into the joint. The histories of some of the reported cases would appear to confirm this. Among the accounts in the literature of cases of suppurative sacroiliitis, a number occurred shortly after childbirth or abortion. This case appears to be the only one occurring antenatally.
Case no. 5. J.S. aged 22 years. The patient was 36 weeks pregnant. It was her first pregnancy which has proceeded normally to date. She was admitted complaining of backache associated with malaise of four to five days duration. The symptoms had become more severe during the preceding twenty-four hours and the pain had moved into the buttock with radiation down the back of the thigh, the outer aspect of the calf and lateral side of the foot.

On examination, she looked ill with a temperature of $39.2^{\circ} \mathrm{C}$. Although the sciatic pain was very severe, the straight leg raising was normal and there were no abnormal neurological signs in the legs.

Investigations revealed a white cell count of $25,000 / \mathrm{cu}$. mm. with a polymorphonucleocytosis. Intramuscular penicillin was commenced after a blood culture had been taken. In the next forty-eight hours the pain became more severe, there being localised tenderness over the sacro-iliac joint. The temperature remained high.

At this time the decision was taken to aspirate the joint and simultaneously perform a Caesarian section. The joint contained a large amount of pus which on culture grew a coagulase positive staphylococcus pyogenes as did the blood culture. As a result of the culture sensitivities, erythromycin being the drug of choice, was commenced. Subsequently the patient made a rapid recovery and her baby thrived. A radiograph taken three weeks later showed an area of sclerosis in the ilium just anterior to the sacro-iliac joint which was regarded as the initial focus of infection. A year later the joint had spontaneously fused.

\section{Discussion}

The classification of osteomyelitis of the pelvis into clinical syndromes clarifies a condition of recognised diagnostic difficulty. Osteomyelitis of the spine presents a similar problem, and Puig (1946) clarified diagnosis in a like manner, with his classification into meningeal, abdominal and hip syndromes.

The clinical features in patients with osteomyelitis of the pelvis vary to such an extent that they may be referred to one of several hospital departments, e.g. general medicine (septicaemic syndrome), general surgery (abdominal syndrome), paediatric (limping child), or even orthopaedic. The incidence of this disease is low and unless pelvic osteomyelitis is borne in mind by the examining doctor the correct diagnosis may be missed during the early stage when the correct treatment would be most beneficial.

As the infective lesion progresses the clinical features multiply and syndromes emerge. Most of our patients had features of two syndromes but one syndrome predominated, preceded the other and was related to the site of the initial focus of infection. Stress has to be laid on these clinical features as early diagnosis is only possible by considering them. Radiographic changes are late in appearing and the 
infection may be well established and involve a large area of bone before radiographic changes are recognisable.

Among the initial routine investigations the white blood cell count was of no diagnostic help, as a raised count was found in only approximately half of our cases. The erythrocyte sedimentation rate was raised in all cases, suggesting that a normal value would be against a diagnosis of acute osteomyelitis. Blood culture was positive in two-thirds of the patients, and the value of isolating and obtaining the sensitivity of the causative organism at an early stage is obvious.

This series illustrates that like osteomyelitis in general, osteomyelitis of the pelvis has a far better prognosis since the advent of antibiotics. Thus, there were no deaths, and spread of the disease to the opposite side of the pelvis which Butler (1940) describes as common, was not seen. However, extensive bony destruction and permanent disability is not infrequent and affects the hip joint in particular, three of our patients eventually having a fused hip (Fig. 4).

\section{Summary}

From study of 18 cases of osteomyelitis of the pelvic bones, the modes of presentation and difficulties of diagnosis are discussed. A classification is suggested based upon this study. The presentation may be septicaemia or in one of four clinical syndromes. These may merge to a greater or lesser degree.

The literature on the subject is reviewed, the incidence of the condition being similar in this series to that noted by other authors.

That the majority of cases occur in the ilium is confirmed, the reason for this lying not only in its bigger volume, but also in its greater vascularity.

As the condition may present in one of many specialist departments it should be borne in mind in cases of unexplained septicaemia.

It is a pleasure to acknowledge the help and encouragement of Mr. W. J. W. Sharrard in the preparation of this paper.

The cases were under the care of Mr. Sharrard, Mr. F. W. Holdsworth and Mr. D. K. Evans to whom we are also indebted.

\section{REFERENCES}

Avila, Leon JR. (1941): Primary Pyogenic Infection of the Sacno-Iliac Articulation, J. Bone Jt Surg., 23, 922.

BeARSE, C. (1923): Osteomyelitis of the Ilium in Children, J. Amer. med. Ass., 80, 991.

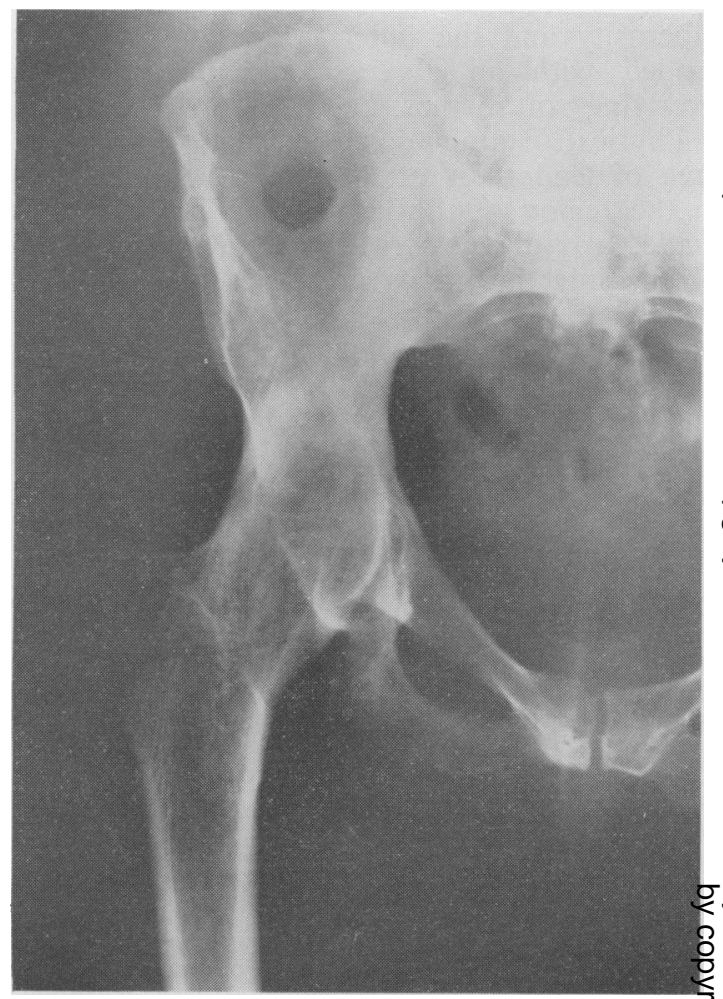

FIG. 4.-Late result of osteomyelitis of the pelve treated in 1948. The hip is fused with $1 \frac{1}{2}$ inches of true shortening. A sequestrum of the ilium has lefit a punched out defect in its blade.

Bruckner, H. (1952): Der Perityphlitische Symptemenkomplex bei re: Beckenschaufelosteomyelitis verursacht durch Pseudo-appendicitis (lokale Kontaktperitonitis) unde echte Begleitappendicitis, Bruns'. Beitr. klin. Chir., 184, 359.

Buosanti, P. (1924): L'Osteomielite acuta dell' ileo nell' infanzia, Arch. ital. Chir., 10, 1.

Butler, E. C. B. (1940): The Treatment, Complications and Late Results of Acute Haematogenous Osteomyelitis, Brit. J. Surg., 28, 261.

FLICKINGER, W. G. (1927): Osteomyelitis of Ilium, Long. Is. med. J., 21, 95.

FROENER, E. (1889): Beitrage zur Kenntnis der akuten spontanen Osteomyelitis der Kurzen und platten Knochen, Bruns'. Beitr. klin. Chir., 5, 79.

KRASNOBAJIV (1925): Nov. khir. Arkh., 10, 354.

Puig, G. (1946): Pyogenic Osteomyelitis of llium, J. Bone Jt Surg., 28, 29.

SHORT, A., RENDLE (1931): Acute Osteomyelitis of Ilium, Brit. med. J., ii, 97.

Simmons, C. C. (1915): The Treatment of Osteomyelitis, Surg. Gynec. Obstet., 20, 129.

VON BERGMAN, A. (1906): Erfahrungen ueber Beckenosteo-myelitis, Arch. klin. Chir., 80, 504.

WELD, P. W. (1960): Osteomyelitis of the Ilium masquerading as Acute Appendicitis, J. Amer. med. Ass., 173, 634.

YounG, F. (1934): Acute Osteomyelitis of the Ilium, Surg. Gynec. Obstet., 58, 986. 